\title{
The effects of telework satisfaction and Zoom fatigue on mental health: a pilot study
}

Heydi Martinez Blandin ${ }^{1}$, Charity Pagoaga Sarres ${ }^{2}$, Óscar Munguía Espinoza ${ }^{3}$, Ernesto Gálvez Pineda ${ }^{4}$, and Miguel Landa-Blanco ${ }^{5 *}$

National Autonomous University of Honduras

${ }^{1}$ Master's degree in Clinical Psychology, School of Psychological Sciences, National Autonomous University of Honduras. ORCID: 0000-0002-0345-3641

${ }^{2}$ Master's degree in Clinical Psychology, School of Psychological Sciences, National Autonomous University of Honduras. ORCID: 0000-0002-5406-1517

${ }^{3}$ School of Psychological Sciences, National Autonomous University of Honduras. ORCID: 0000-0002-6072-5316

${ }^{4}$ Master's degree in Clinical Psychology, School of Psychological Sciences, National Autonomous University of Honduras. ORCID: 0000-0001-6395-4037

${ }^{5}$ Master's degree in Clinical Psychology, School of Psychological Sciences, National Autonomous University of Honduras. ORCID: 0000-0002-7865-7593

*Correspondence regarding the manuscript should be sent to Miguel Landa-Blanco, miguel.landa@unah.edu.hn 


\begin{abstract}
The current study shows how symptoms of somatization, depression, and anxiety were affected by telework satisfaction and Zoom fatigue. This was made through a quantitative approach in a sample of 199 employees. Somatization, depression, and anxiety symptoms were measured through the BSI-18. The Zoom Exhaustion \& Fatigue Scale (ZEFS) was used to evaluate different forms of videoconferencing fatigue, including visual, social, motivational, emotional, and general fatigue. The Work Satisfaction for Teleworkers Questionnaire (WSTQ) was used to measure telework satisfaction. Results indicate that somatization symptoms were significantly predicted by ZEFS-visual fatigue, ZEFS-motivational fatigue, and age. ZEFS-emotional fatigue and respondent's age, predicted depression symptoms. Anxiety scores were significantly predicted by ZEFS-emotional fatigue. When considering ZEFS, respondents' age, sex, and years working at the institution, satisfaction with telework was not related to any mental health indicators. All regression models had large effect sizes. Results suggest that videoconferencing fatigue can have direct repercussions on workers' mental health. The findings are discussed, considering previous research and practical implications.
\end{abstract}

Keywords: Zoom fatigue, mental health, telework, anxiety, somatization, depression 


\section{Introduction}

In December 2019, the first suspicious cases of COVID-19 were detected in Wuhan, China. Due to its epidemiological characteristics, by March of 2020, the COVID-19 outbreak was characterized as a pandemic, being the fifth documented pandemic since the $1918 \mathrm{flu}$ pandemic. This virus has a highly contagious scope and continuously evolves among the human population (Liu et al., 2020). Given that COVID-19 is rapidly transmitted from human to human, social distancing measures have been implemented worldwide to control the spread of the virus. Specific social distancing strategies include school closure, lockdowns, and remote work, changing the labor dynamic drastically (Nande et al., 2021).

Therefore, many public and private institutions worldwide had to change their labor policies and normalize telework as an alternative to in-situ work. Moreover, although there are arguments regarding the adaptation of telework conditions that can have different economic and social benefits (Abulibdeh, 2020), there have been unexpected side effects on workers worldwide that deserve attention. In many cases, telework relies heavily on videoconferencing activities, and this has brought different apparent repercussions.

One of the most critical side effects due to the exhaustion related to videoconferencing has been Zoom Fatigue, as it has been popularly called. This phenomenon can be experienced in several domains, including emotional, visual, motivational, and social fatigue (Fauville et al., 2021). Some videoconference stressors users report include meeting duration, lack of structure, impunctuality, and seeing other participants multi-tasking during the meetings (Karl et al., 2021).

There are different potential explanations for videoconferencing fatigue, including a specific type of anxiety referred to as Mirror Anxiety. This usually happens when people are in front of their reflection, they become self-aware and tend to self-evaluate their reflection. In videoconferencing, the user constantly observes their image on the screen; this can relate to prolonged scrutiny of their appearance and behavior in front of the camera, resulting in a stressful event (Bailenson, 2021). Recent studies have concluded that emotional exhaustion has significant adverse effects on employees' satisfaction with work (Prajogo, 2019).

Considering the facts above, the purpose of this current study was to analyze the effects that telework satisfaction and Zoom exhaustion and fatigue have over mental health indicators, 
specifically, somatization, anxiety, and depression symptoms. This study was accomplished during the COVID-19 pandemic through a sample of Honduran workers. This exploratory-pilot study can serve as a foundation for future research on the interaction between mental health and digital work environments.

\section{Materials and Methods}

\section{Participants}

A non-probabilistic, snow-ball sampling was made to collect data. The online survey was distributed through social media and the work-related websites of Honduras. A total of 199 participants responded the survey, $64.32 \%(n=128)$ were female and $35.68 \%(n=71)$ male. Their mean age was of 34.77 years $(S D=10.70)$. Most respondents $(n=131 ; 65.83 \%)$ did full-time telework, and the remaining $34.17 \%(n=68)$ did a combination of telework and in-office work.

\section{Instruments}

\section{Work Satisfaction for Teleworkers Questionnaire}

The Work Satisfaction for Teleworkers Questionnaire (WSTQ) is a 47-item Likert-type scale (Hernández Salazar, 2019), with a five-point response system (1=completely unsatisfied, $5=$ completely satisfied). It measures satisfaction with the following domains: work conditions, supervision, acknowledgments, transcendence, independence-autonomy, communication with other areas, career development, available technology, job performance, work-life balance, health, economy, and psychosocial risk. In our study, the WSTQ achieved an overall internal consistency of 0.96. Some items included: "How satisfied are you with the assignments and activities you have to do as part of your telework?"; "How satisfied are you with the lighting conditions in your telework area?", among other items.

\section{Zoom Exhaustion \& Fatigue Scale}

The Zoom Exhaustion \& Fatigue Scale (ZEFS) consists of 15 items, with a five-point Likert type scale response set (1=not at all, 5=extremely). The ZEFS describes several types of 
fatigue, including general ( $\alpha=0.93)$, visual $(\alpha=0.91)$, social $(\alpha=0.89)$, motivational $(\alpha=0.89)$, emotional $(\alpha=0.80)$, with an overall consistency acceptable internal consistency $(\alpha=0.94)$, see Table 1. Some items included: "How tired do you feel after video conferencing?", "How much do your eyes hurt after video conferencing?", among others.

Table 1

Internal consistency of the Zoom Exhaustion \& Fatigue Scale

\begin{tabular}{cccccccc}
\hline Scale & Subscale & Mean & $S D$ & $\alpha$ & $L L$ & $U L$ & Items \\
\hline \multirow{4}{*}{ ZEFS } & General fatigue & 10.698 & 3.145 & 0.93 & 0.92 & 0.95 & 3 \\
& Visual fatigue & 10.015 & 3.524 & 0.91 & 0.89 & 0.93 & 3 \\
& Social fatigue & 9.487 & 3.752 & 0.89 & 0.86 & 0.92 & 3 \\
& Motivational & & & & & & \\
& fatigue & 9.387 & 3.036 & 0.80 & 0.76 & 0.85 & 3 \\
& Emotional fatigue & 9.090 & 3.524 & 0.91 & 0.88 & 0.93 & 3 \\
& ZEFS Total & 48.678 & 13.993 & 0.94 & 0.93 & 0.95 & 15 \\
\hline
\end{tabular}

Note. Intervals were calculated at a 95\% Confidence Level. Higher scores in the ZEFS indicate higher self-reported fatigue.

\section{Brief Symptom Inventory-18}

Mental health indicators were measured using the Brief Symptom Inventory-18 (BSI-18), an 18-item scale that measures the prevalence and intensity of symptoms of depression, anxiety, and somatization ( $\mathrm{Li}$ et al., 2018). Each item is rated on a five-point Likert type scale (1=Not at all, 5=All the time). Based on our data, the total BSI-18 had an adequate internal consistency $(\alpha=0.95)$, as well as each of its subscales: somatization $(\alpha=0.87)$, depression $(\alpha=0.88)$, and anxiety $(\alpha=0.88)$, see Table 5 . 


\section{Table 5}

Internal consistency of the Brief Symptom Inventory-18

\begin{tabular}{llcccc}
\hline Scale & Subscale & $\alpha$ & $L L$ & $U L$ & Items \\
\hline \multirow{4}{*}{ BSI } & Somatization & 0.87 & 0.84 & 0.9 & 6 \\
& Depression & 0.88 & 0.86 & 0.91 & 6 \\
& Anxiety & 0.88 & 0.85 & 0.91 & 6 \\
& BSI Total & 0.95 & 0.94 & 0.96 & 18 \\
\hline
\end{tabular}

Note. Intervals were calculated at a 95\% Confidence Level.

\section{General information questionnaire}

The online survey also collected data regarding the respondents' sex, age, self-reported hours of telework per week, and years the employee has worked in the institution.

\section{Data analysis}

First, the reliability of each questionnaire was determined through Cronbach's alpha. Then, summative totals and descriptive statistics were obtained for each questionnaire. As all questions were required, no missing data were present in the data set. Later, three separate linear multiple regression models were built for each mental health outcome included in the study (somatization, depression, and anxiety).

All models included the following predictors: ZEFS subscales (General fatigue, Visual fatigue, Social fatigue, Motivational fatigue, Emotional fatigue), Work Satisfaction for Teleworkers Questionnaire scores, age, sex (female=1, male=0), hours of telework per week, and years working in the institution. The significance of the coefficients was established at a 95\% confidence interval. Later, effect sizes and power indicators were determined for each model, $f^{2}$ values were classified as follows: small (.02-.14), medium (.15-.34), and large (>.35) (Cohen, 1992). 


\section{Ethical considerations}

The present study was designed following the ethical guidelines required by the master's degree in Clinical Psychology of the National Autonomous University of Honduras. All potential participants were asked to read and accept an Informed Consent form which detailed the purpose of the study, the anonymity of the data collection process, the potential risks, and the benefits of the study. Accepting the conditions stated in the Informed Consent form was a requirement for participants to be included in the study.

\section{Results}

\section{Somatization symptoms}

The average score for the somatization subscale was $6.477(S D=6.006)$. A linear regression model was used to determine the variables that significantly affected somatization scores; the model achieved an $r^{2}$ of $0.334, F(10,186)=9.326, p<.001$. Significant predictors included ZEFS-visual fatigue $(\beta=0.233 ; p=0.006)$, ZEFS-motivational fatigue $(\beta=0.297$; $p=0.043)$, ZEFS-emotional fatigue $(\beta=0.297 ; p=0.005)$ and respondent's age $(\beta=-0.192$; $p=0.045)$. ZEFS-general fatigue, ZEFS-social fatigue, satisfaction with telework, respondent's sex, hours of telework per week, and years working in the institution were not significant predictors $(p>0.05)$; see Table 2 . The overall model achieved a large effect size $\left(f^{2}=0.501\right)$, with high power (0.999).

Table 2

Regression model explaining somatization symptoms

\begin{tabular}{clccccc}
\hline Model & Predictors & Unstandardized & SE & Standardized & $t$ & $p$ \\
\hline $\mathrm{H}_{\mathbf{0}}$ & (Intercept) & 6.503 & 0.43 & & 15.135 & $<.001$ \\
\hline \multirow{2}{*}{} & (Intercept) & -2.756 & 3.462 & & -0.796 & 0.427 \\
& ZEFS & & & & & \\
$\mathrm{H}_{1}$ & General fatigue & -0.033 & 0.18 & -0.017 & -0.18 & 0.857 \\
& Visual fatigue & 0.399 & 0.143 & 0.233 & 2.789 & $\mathbf{0 . 0 0 6}$ \\
& Social fatigue & -0.139 & 0.141 & -0.086 & -0.983 & 0.327 \\
\hline
\end{tabular}




\begin{tabular}{lccccc}
\hline \multicolumn{1}{c}{ Motivational fatigue } & 0.381 & 0.187 & 0.193 & 2.042 & $\mathbf{0 . 0 4 3}$ \\
$\quad$ Emotional fatigue & 0.508 & 0.181 & 0.297 & 2.81 & $\mathbf{0 . 0 0 5}$ \\
WSTQ & 0.303 & 0.715 & 0.028 & 0.425 & 0.672 \\
Age & -0.108 & 0.053 & -0.192 & -2.022 & $\mathbf{0 . 0 4 5}$ \\
Sex & 0.875 & 0.816 & 0.07 & 1.072 & 0.285 \\
$\begin{array}{l}\text { Hours of telework per } \\
\text { week }\end{array}$ & 0.01 & 0.018 & 0.035 & 0.563 & 0.574 \\
$\begin{array}{l}\text { Years working in the } \\
\text { institution }\end{array}$ & & & & & \\
\end{tabular}

Note. Significant p-values (<.05) are presented in bold case letters.

\section{Depression symptoms}

The average score for the depression subscale was 6.734 ( $S D=6.256)$. The regression model achieved an $r^{2}$ of $0.351, F(10,186)=10.080, p<.001$. ZEFS-emotional fatigue $(\beta=0.334$; $p=0.002)$ and respondent's age $(\beta=-0.213 ; p=0.024)$ were the only significant predictor of depressive symptoms scores; see Table 3. The overall model achieved a large effect size $\left(f^{2}=0.540\right)$, with high power $(0.999)$.

Table 3

Regression model explaining depression symptoms

\begin{tabular}{llccccc}
\hline Model & Predictors & Unstandardized & SE & Standardized & $t$ & $p$ \\
\hline $\mathrm{H}_{0}$ & (Intercept) & 6.751 & 0.448 & & 15.078 & $<.001$ \\
\hline \multirow{2}{*}{ (Intercept) } & 2.346 & 3.561 & & 0.659 & 0.511 \\
& ZEFS & & & & & \\
& General fatigue & 0.022 & 0.185 & 0.011 & 0.12 & 0.904 \\
$\mathrm{H}_{1} \quad$ Visual fatigue & 0.028 & 0.147 & 0.016 & 0.191 & 0.849 \\
& Social fatigue & 0.153 & 0.145 & 0.091 & 1.054 & 0.293 \\
& Motivational fatigue & 0.311 & 0.192 & 0.151 & 1.618 & 0.107 \\
& Emotional fatigue & 0.594 & 0.186 & 0.334 & 3.195 & $\mathbf{0 . 0 0 2}$
\end{tabular}




\begin{tabular}{lccccc}
\hline WSTQ & -0.794 & 0.735 & -0.07 & -1.081 & 0.281 \\
Age & -0.125 & 0.055 & -0.213 & -2.273 & $\mathbf{0 . 0 2 4}$ \\
Sex & -0.164 & 0.839 & -0.013 & -0.195 & 0.845 \\
Hours of telework per & 0.010 & 0.019 & 0.032 & 0.517 & 0.606 \\
week & & & & & \\
$\begin{array}{l}\text { Years working in the } \\
\text { institution }\end{array}$ & 0.079 & 0.068 & 0.107 & 1.16 & 0.248 \\
\hline
\end{tabular}

Note. Significant p-values (<.05) are presented in bold case letters.

\section{Anxiety symptoms}

The average score for the anxiety subscale was $6.673(S D=5.979)$. The model explaining anxiety symptoms scores achieved an $r^{2}$ of $0.353, F(10,186)=10.133, p<.001$. ZEFS-emotional fatigue $(\beta=0.300 ; p=0.002)$ was the only individual significant predictor of anxiety symptoms; all remaining variables yielded non-significant coefficients $(p>0.05)$. The overall model achieved a large effect size $\left(f^{2}=0.545\right)$, with high power $(0.999)$, see Table 4.

Table 4

Regression model explaining anxiety symptoms

\begin{tabular}{llccccc}
\hline Model & Predictors & Unstandardized & SE & Standardized & $t$ & $p$ \\
\hline $\mathrm{H}_{0}$ & (Intercept) & 6.695 & 0.428 & & 15.649 & $<.001$ \\
\hline \multirow{2}{*}{ (Intercept) } & -1.517 & 3.399 & & -0.446 & 0.656 \\
& ZEFS & & & & & \\
& General fatigue & 0.028 & 0.177 & 0.015 & 0.159 & 0.874 \\
& Visual fatigue & 0.263 & 0.14 & 0.154 & 1.872 & 0.063 \\
& Social fatigue & -0.013 & 0.138 & -0.008 & -0.096 & 0.923 \\
$\mathrm{H}_{1}$ & Motivational fatigue & 0.325 & 0.183 & 0.165 & 1.776 & 0.077 \\
& Emotional fatigue & 0.510 & 0.177 & 0.300 & 2.874 & $\mathbf{0 . 0 0 5}$ \\
& WSTQ & -0.141 & 0.702 & -0.013 & -0.201 & 0.841 \\
& Age & -0.081 & 0.052 & -0.144 & -1.54 & 0.125 \\
& Sex & 1.152 & 0.801 & 0.092 & 1.437 & 0.152
\end{tabular}




\begin{tabular}{|c|c|c|c|c|c|}
\hline Hours of telework per & & & & & \\
\hline week & 0.001 & 0.018 & 0.002 & 0.038 & 0.970 \\
\hline Years working in the & & & & & \\
\hline institution & 0.026 & 0.065 & 0.036 & 0.393 & 0.695 \\
\hline
\end{tabular}

Note. Significant p-values (<.05) are presented in bold case letters.

\section{Discussion}

The results of this research suggest that Zoom Fatigue has direct effects on workers' mental health. The fact that Visual and Motivational Fatigue are significant predictors of somatization symptoms represents an essential indicator of this analysis. Notably, Depression symptoms were predicted by Emotional Fatigue, and anxiety scores were significantly predicted by Emotional Fatigue. When considering Zoom Fatigue, demographic, and work-related variables, telework satisfaction did not significantly affect any mental health indicators included in the study.

Mandatory remote working impacts the employees' home environment on a social, financial, and logistical level. As such, some research recommends that organizations compensate their remote employees for such an additional burden to lessen its impact on their output and lifestyle (Oakman et al., 2020).

Due to the frequency in the use of remote communication applications, together with the effectiveness they have proven to have during the pandemic, videoconferencing and teleworking are here to stay. Organizations must regulate their use and know the implications that this modality of work and teleconferencing tools have on the worker's health. In this sense, one of the elements that companies must consider is the time spent on videoconferences and try to have a maximum duration of one hour. Since there is evidence of a relationship between the duration of meetings using a web-based platform and general wellbeing. Consequently, long meetings (two or more hours) may result in a marked deterioration of employees' wellbeing compared to shorter, one-hour meetings (Kershaw et al., 2021).

On the other hand, it is crucial to consider that training and familiarity with these videoconferencing tools reduce the stress associated with their use. They strengthen the 
resources that the employee has to carry out their work, impacting their general wellbeing. Likewise, training in teleworking performance monitoring and evaluation strategies for managers and administrators or managers can reduce the need to schedule frequent meetings with the worker, as other indicators are used to measure performance (Kershaw et al., 2021).

Despite the relevance of the findings, this study is not without limitations. For instance, the limited sample size and non-probabilistic selection procedure may limit the generalization of the results. On the other hand, no data was collected regarding meetings duration or frequency. Future studies should overcome these limitations and include qualitative methodologies to understand further Zoom Fatigue and its effects on everyday life and productivity. Studies should also explore the relationship between telework, Zoom Fatigue and burnout, and how these variables affect workers' personal and social wellbeing. Further studies should also focus on whether video cameras have differentiated effects on Zoom Fatigue instead of audio-only conferencing.

\section{References}

Abulibdeh, A. (2020). Can COVID-19 mitigation measures promote telework practices? Journal of Labor and Society, 23(4), 551-576. https://doi.org/https://doi.org/10.1111/wusa.12498

Bailenson, J. N. (2021). Nonverbal Overload: A Theoretical Argument for the Causes of Zoom Fatigue. Technology, Mind, and Behavior, 2(1). https://doi.org/10.1037/TMB0000030

Cohen, J. (1992). A power primer. Psychological Bulletin, 112(1), 155-159. https://doi.org/10.1037/0033-2909.112.1.155

Fauville, G., Luo, M., Queiroz, A. C. M., Bailenson, J. N., \& Hancock, J. (2021). Zoom Exhaustion \& Fatigue Scale. Computers in Human Behavior Reports, 4, 100119. https://doi.org/https://doi.org/10.1016/j.chbr.2021.100119

Hernández Salazar, L. V. (2019). Diseño y aplicación de un modelo de medición de satisfacción laboral para teletrabajadores en Colombia. Caso entidad de Salud. https://bdigital.uexternado.edu.co/handle/001/2226

Karl, K. A., Peluchette, J. V, \& Aghakhani, N. (2021). Virtual Work Meetings During the 
COVID-19 Pandemic: The Good, Bad, and Ugly. Small Group Research, 10464964211015286. https://doi.org/10.1177/10464964211015286

Kershaw, M. E., Lupien, S. P., \& Scheid, J. L. (2021). Impact of Web-Based Meeting Platform Usage on Overall Well-Being among Higher Education Employees. European Journal of Investigation in Health, Psychology and Education, 11(2).

https://doi.org/10.3390/ejihpe11020028

Li, M., Wang, M.-C., Shou, Y., Zhong, C., Ren, F., Zhang, X., \& Yang, W. (2018).

Psychometric Properties and Measurement Invariance of the Brief Symptom Inventory-18 Among Chinese Insurance Employees . In Frontiers in Psychology (Vol. 9, p. 519). https://www.frontiersin.org/article/10.3389/fpsyg.2018.00519

Liu, Y.-C., Kuo, R.-L., \& Shih, S.-R. (2020). COVID-19: The first documented coronavirus pandemic in history. Biomedical Journal, 43(4), 328-333. https://doi.org/https://doi.org/10.1016/j.bj.2020.04.007

Nande, A., Adlam, B., Sheen, J., Levy, M. Z., \& Hill, A. L. (2021). Dynamics of COVID-19 under social distancing measures are driven by transmission network structure. PLOS Computational Biology, 17(2), e1008684. https://doi.org/10.1371/journal.pcbi.1008684

Oakman, J., Kinsman, N., Stuckey, R., Graham, M., \& Weale, V. (2020). A rapid review of mental and physical health effects of working at home: how do we optimize health? $B M C$ Public Health, 20(1), 1825. https://doi.org/10.1186/s12889-020-09875-z

Prajogo, W. (2019). The relationship among emotional exhaustion, job satisfaction, performance, and intention to leave. Advances in Management and Applied Economics, 9(1), 21-29. https://ideas.repec.org/a/spt/admaec/v9y2019i1f9_1_2.html 